\title{
Retour d'expérience des attentats du 13 novembre 2015. Rôle spécifique des hôpitaux de proximité
}

\author{
Feedback on Terrorist Attacks on November 13, 2015. Specific Role of the Closest Hospitals
}

\author{
E. Debuc $\cdot$ J.-P. Fontaine $\cdot$ C. Ogereau $\cdot$ C. Tournon $\cdot$ O. Peyrony $\cdot$ P. Berrebi $\cdot$ J. Azuar $\cdot$ O. Gardy $\cdot$ B. Sakarovitch $\cdot$ \\ F. Geffrier $\cdot$ Y. Yordanov $\cdot$ D. Pateron
}

Reçu le 15 décembre 2015 ; accepté le 4 janvier 2016

(C) SFMU et Lavoisier SAS 2016

Résumé Lors de l'attentat terroriste du 13 novembre 2015 à Paris, les hôpitaux de Saint-Antoine et de Saint-Louis qui étaient à proximité des sites impliqués ont été intégrés dans le dispositif plan d'Urgence régulé par le Samu comme hôpitaux de proximité. L'objectif de cet article est de décrire l'activité et le type de malades qui sont arrivés dans ces deux hôpitaux et de présenter les éléments mis en évidence lors des séances de retour d'expérience organisées dans ces établissements. L'alerte a été donnée dans les deux hôpitaux par l'arrivée des premiers blessés par balles. Quarante-cinq patients sont arrivés au service des urgences de l'hôpital Saint-Antoine (6 ont été triés en urgences absolues [UA] et 39 en urgences relatives [UR]) et 26 à l'hôpital Saint-Louis (10 UA, 16 UR). La coopération de l'ensemble des structures impliquées dans la prise en charge des blessés a été un élément fondamental d'efficacité du plan d'Urgence. La solidarité manifestée lors de l'événement ainsi que les compétences professionnelles ont permis un bon fonctionnement de ces services d'urgences. Les difficultés de fonctionnement et des propositions d'amélioration concernent chaque étape de la prise en charge même si celle-ci a été relativement courte, de l'ordre de 12 heures. Les hôpitaux à proximité d'un événement avec de multiples victimes sont sollicités de façon particulière, et chaque hôpital doit s'y

\footnotetext{
E. Debuc $\cdot$ C. Tournon $\cdot$ P. Berrebi $\cdot$ O. Gardy $\cdot$ B. Sakarovitch .

F. Geffrier $\cdot$ Y. Yordanov $\cdot$ D. Pateron $(\bowtie)$

Service des urgences,

centre hospitalier universitaire Saint-Antoine,

Assistance publique-Hôpitaux de Paris (AP-HP),

184, rue du Faubourg Saint-Antoine, F-75012 Paris, France

e-mail : dominique.pateron@aphp.fr
}

Sorbonne universités, UPMC Univ Paris 06, F-75005 Paris

J.-P. Fontaine $\cdot$ C. Ogereau $\cdot$ O. Peyrony $\cdot$ J. Azuar

Service des urgences, centre hospitalier universitaire Saint-Louis,

AP-HP, 1, avenue Claude-Vellefaux, F-75010 Paris, France préparer. La régulation médicalisée du Samu doit éviter d'adresser des malades UA vers les établissements de proximité.

Mots clés Attentat $\cdot$ Plaie par balle $\cdot$ Service d'urgences · Tri $\cdot$ Retour d'expérience $\cdot$ Urgences absolues

\begin{abstract}
During the terrorist attack on 13 November 2015 in Paris, Saint-Antoine and Saint-Louis Hospitals, which were in close proximity to the areas involved, were included in the Emergency Plan overseen by the French Emergency Medical Services (Samu), as nearby hospitals. The aim of this article is to describe the activity and the type of patients who came into these two hospitals and present the areas highlighted during the feedback sessions organised in these establishments. The alarm was raised in both hospitals through the arrival of the first gunshot victims. Forty-five patients were brought to the Emergency department of Saint-Antoine Hospital (6 were classified as absolute emergencies [Aes] and 39 as relative emergencies [Res]) and 26 to Saint-Louis Hospital (10 as AEs and 16 as REs). The cooperation of all of the organisations involved in treating the casualties was a fundamental part of the success of the emergency plan. The solidarity demonstrated during the event, as well as the professional expertise enabled these emergency departments to operate effectively. Operational challenges and suggested improvements affect each stage of the treatment process, even if it was only relatively short, i.e. around 12 hours. Hospitals close to an event with multiple victims are called upon in a specific way, and each hospital must be prepared for such an eventuality. French Emergency Medical Services (Samu) dispatch rules should avoid sending AE patients to nearby hospitals.
\end{abstract}

Keywords Attack - Gunshot wound - Emergency services · Triage $\cdot$ Feedback $\cdot$ Absolute emergencies 


\section{Introduction}

La circulaire du 3 mai 2002 impose la rédaction d'un Plan Blanc à tous les établissements de santé disposant d'un service d'accueil des urgences [1]. Le Plan Blanc de l'Assistance publique des Hôpitaux de Paris (AP-HP) regroupe les dispositions que chaque établissement de cette institution doit prendre lorsqu'un événement risque d'aboutir à un afflux massif de victimes. Dans une telle éventualité, les hôpitaux à proximité de l'événement tiennent une place particulière dans le dispositif [2]. Le recours à leurs services d'urgences (SU) peut être important de façon spontanée, et la répartition des victimes régulées par le Samu doit donc tenir compte de cette réalité. Le vendredi 13 novembre 2015, l'AP-HP est alertée à $21 \mathrm{~h} 40$ que des fusillades, dans le cadre d'une probable attaque terroriste multisite, ont lieu dans différents cafés des $11^{\mathrm{e}}$ et $10^{\mathrm{e}}$ arrondissements de Paris. Une attaque, impliquant plusieurs centaines de personnes, a lieu également au Bataclan ( $11^{\mathrm{e}}$ arrondissement). Il apparaît alors clairement que les deux hôpitaux de proximité, à savoir l'hôpital Saint-Louis et l'hôpital Saint-Antoine, risquent d'être fortement impactés. Trois des cinq cafés où ont eu lieu les fusillades sont situés respectivement à 200, 250 et 700 mètres de l'hôpital Saint-Louis et deux sont situés à 500 mètres et à 800 mètres de l'hôpital Saint-Antoine (Fig. 1). Enfin, le Bataclan, lieu de spectacle accueillant plus de mille personnes est respectivement à $1,4 \mathrm{~km}$ de l'hôpital Saint-Louis et à 1,8 km de l'hôpital Saint-Antoine.

Dans cet article, notre objectif est double : décrire l'activité et le type de malades qui sont arrivés dans ces deux hôpitaux, situés à proximité des fusillades et présenter les éléments mis en évidence lors des séances de retour d'expérience organisées dans ces établissements, au niveau des services des urgences, pour analyser les éventuels problèmes de fonctionnement ou nécessités repérées au cours de la nuit du

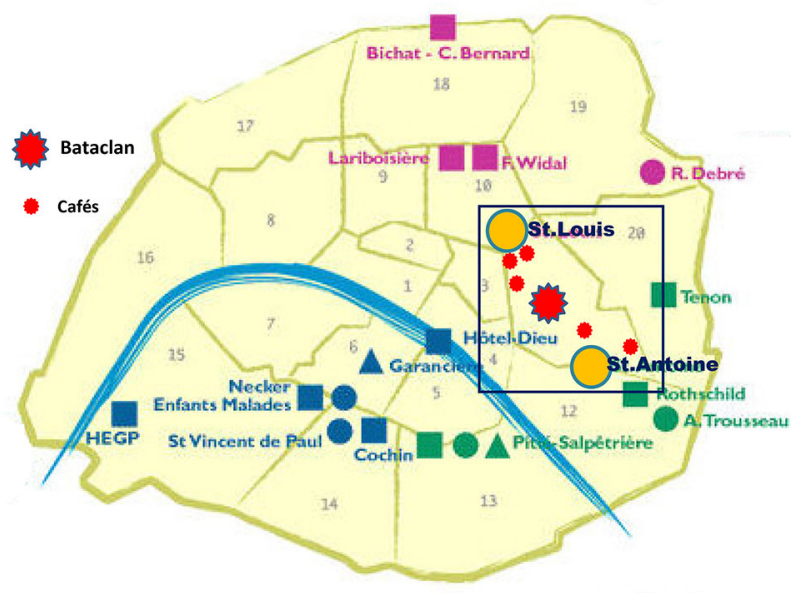

Fig. 1 Carte de Paris avec les lieux des attentats par rapport aux deux hôpitaux de proximité
13 au 14 novembre et enfin dégager les pistes d'amélioration dans la mise en place du dispositif d'un hôpital en situation de proximité d'un tel événement.

\section{Méthodes}

Un recueil de données concernant le profil des patients victimes de l'attaque terroriste, en particulier les blessés par balles et leur prise en charge sur ces deux hôpitaux a été effectué.

Les éléments repérés et les propositions d'amélioration dégagées lors des retours d'expérience sont présentés sous forme de binômes. Ces points ne sont pas exhaustifs et ne prétendent pas représenter l'ensemble des difficultés de fonctionnement rencontrées mais correspondent aux éléments mis en avant lors des séances de retour d'expérience. Certains de ces points font partie du Plan Blanc prévu mais sont indiqués lorsqu'ils ont été ciblés comme une source de difficulté ou un élément d'aide important à la prise en charge des malades.

\section{Activité habituelle et fonctionnement des deux sites avant le déclenchement des attentats}

L'hôpital Saint-Antoine et l'hôpital Saint-Louis reçoivent respectivement 60000 et 37000 urgences adultes par an avec un taux d'hospitalisation de 22 et $15 \%$. Ces deux hôpitaux disposent entre autres d'un service d'imagerie ouvert 24 heures/24, d'un service de chirurgie viscérale, d'une réanimation chirurgicale et d'une réanimation médicale. SaintAntoine dispose d'un service d'orthopédie-traumatologie, ce qui n'est pas le cas de Saint-Louis qui dispose en revanche d'un service d'urologie, d'un service de chirurgie maxillofaciale et d'un service de chirurgie plastique et reconstructrice. Aucun des deux sites ne dispose de service de chirurgie thoracique ou vasculaire, ni de neurochirurgie. Aucun de ces deux sites ne correspond à un site d'accueil des traumatisés graves récemment labellisés par l'Agence régionale de santé (ARS). La situation au sein des urgences au moment de l'événement était conforme à l'activité de chacun des sites pour un vendredi soir :

- à Saint-Antoine : présence de 59 malades sur le site dont sept en attente d'hospitalisation et l'occupation de six lits sur 11 en unité d'hospitalisation de courte durée (UHCD) ;

- sur Saint-Louis : présence de 24 patients dont huit en attente d'hospitalisation et de cinq patients dans les sept lits d'UHCD. 
La situation du SU en termes de présence de patients lors des journées du 13 et du 14 novembre est indiquée dans la Figure 2.

\section{Déclenchement de l'alerte}

À l'hôpital Saint-Louis, l'alerte est donnée par un médecin présent sur les lieux de la première fusillade à $250 \mathrm{~m}$ de l'entrée du site, cafés « le petit Cambodge » et « le Carillon », suivie par l'arrivée des premiers patients dont le premier est enregistré à $21 \mathrm{~h} 42$. À l'hôpital Saint-Antoine, le déclenchement de l'alerte s'est fait par l'arrivée des premiers malades, à savoir trois patients issus du café « la belle équipe » situé à 500 mètres, dont le premier est arrivé à $21 \mathrm{~h} 47$. Dans chacun des deux sites, les premiers patients ont été amenés spontanément par des tierces personnes et par des moyens de transport divers (taxis, voitures personnelles, voitures de tourisme avec chauffeur...).

\section{Activités et type de patients}

De $21 \mathrm{~h} 47$ le 13 novembre à $3 \mathrm{~h} 38$ le 14 novembre, 45 patients blessés par balles sont arrivés au SU de l'hôpital SaintAntoine et 26 à l'hôpital Saint-Louis. Parmi eux, six ont été triés en urgences absolues (UA) et 39 en urgences relatives (UR) sur Saint-Antoine et 11 UA, 15 UR sur SaintLouis. Les patients étaient amenés parfois à plusieurs dans le même véhicule. Les principales caractéristiques concernant le sexe, l'âge, le mode de transport, l'orientation à l'issue du passage des urgences et le devenir sont indiquées dans le Tableau 1. Les 71 patients reçus dans les deux hôpi-

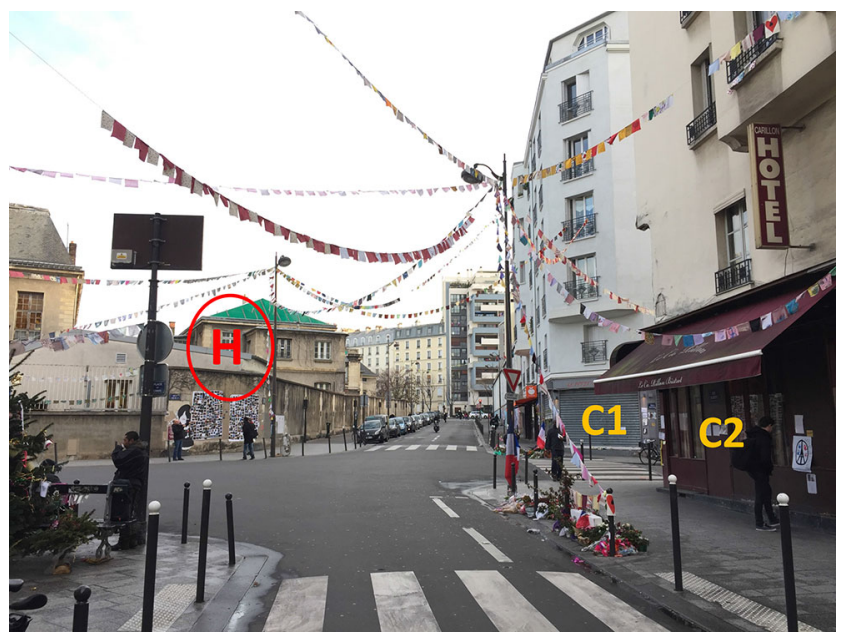

Fig. 2 Illustration de la proximité des cafés « Le Petit Cambodge » (C1) et « Le Carillon » (C2) par rapport à l'enceinte de l'hôpital Saint-Louis (H)
Tableau 1 Caractéristique des victimes vues aux urgences des hôpitaux de proximité.

\begin{tabular}{|lll|}
\hline Hôpital & $\begin{array}{l}\text { Saint- } \\
\text { Louis }\end{array}$ & $\begin{array}{l}\text { Saint- } \\
\text { Antoine }\end{array}$ \\
\hline Nombre de blessés & 26 & 45 \\
Hommes, $n$ (\%) & $16(61)$ & $26(57)$ \\
Femmes, $n(\%)$ & $10(39)$ & $19(43)$ \\
Âge (ans) & $32 \pm 10$ & $35 \pm 10$ \\
Mode d'arrivée & & \\
$\quad$ Médicalisé (UMH) & 0 & 6 \\
$\quad \begin{array}{l}\text { Premier secours non médicalisé } \\
\text { Spontané }\end{array}$ & 20 & 36 \\
Orientation & 6 & 3 \\
$\quad \begin{array}{l}\text { Bloc opératoire ou réanimation } \\
\text { Hospitalisation }\end{array}$ & 9 & 9 \\
$\quad$ Chirurgie ambulatoire ou retour & 3 & 20 \\
$\quad$ à domicile & 15 \\
\hline $\begin{array}{l}\text { Les données sont exprimées en moyenne } \pm \text { écart-type ou nombre } \\
\text { (\%). UMH : unité mobile hospitalière. }\end{array}$ \\
\hline
\end{tabular}

taux de proximité représentent environ un quart des patients blessés par balles transportés dans les 14 hôpitaux de destination primaire. Deux vagues successives peuvent être décrites, une première en rapport avec les premières fusillades en terrasse, une seconde en rapport avec les événements liés au Bataclan.

\section{Organisation initiale (phase suraiguë)}

Dès l'arrivée des premières victimes, les médecins urgentistes présents sur place ont mis en œuvre spontanément les éléments principaux d'un Plan Blanc en situation d'afflux de victime :

- contact avec la direction du site et des responsables du $\mathrm{SU}$;

- prises en charge des premières victimes par les équipes présentes dans les SAU et mobilisation immédiates des ressources internes présentes dans l'hôpital (IDE, chirurgiens digestifs sur les deux sites, orthopédistes de Saint-Antoine et plasticiens à Saint-Louis, anesthésistesréanimateurs, aide administrative pour l'enregistrement des patients);

- organisation d'une première phase de triage à l'accueil permettant d'orienter au plus vite les patients vers une prise en charge chirurgicale immédiate ou une stabilisation/évaluation en salle d'accueil des urgences vitales (SAUV-Saint-Antoine) et/ou en salle de surveillance postinterventionnelle (salle de surveillance postinterventionnelle (SSPI)-Saint-Louis) Louis (Fig. 3) ; 


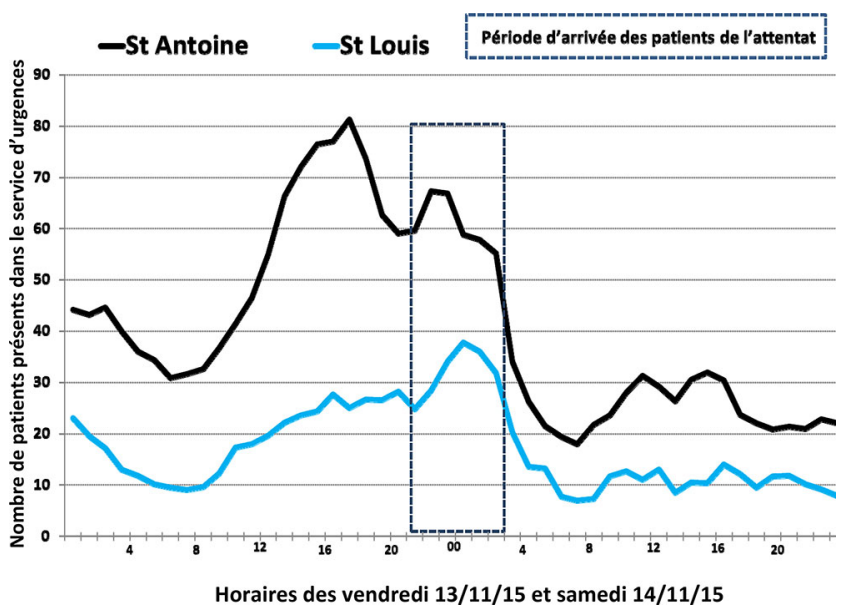

Fig. 3 Nombre de patients présents aux urgences les 13 et 14 novembre 2015 sur les deux sites des urgences de proximité (source : direction de l'organisation médicale et des relations avec l'université de l'AP-HP)

- délégation de la prise en charge des patients « hors attentats » par les équipes médicales mobilisables sur place (hématologue de garde à Saint-Louis, cardiologue, neurologue, puis médecin de la policlinique à Saint-Antoine) ;

- libération de lits des UHCD et passage rapide en salle des patients « hors attentats » en attente d'hospitalisation avec l'aide de l'encadrement.

Cette période initiale s'est déroulée sans information sur la nature et l'évolution des événements, du nombre de victimes potentielles et des possibilités éventuelles de transfert de patients critiques si nécessaire, imposant dans cette situation à chacun des deux sites de fonctionner en autarcie dans un contexte de black-out.

\section{Organisation du renfort et développement du dispositif}

Sur les deux sites, la mobilisation de ressources internes a très vite été accompagnée d'un retour spontané de personnels médicaux et paramédicaux dont beaucoup venaient de quitter leur service ou étaient à proximité de leur hôpital. Enfin, d'autres personnels ont été rappelés par leurs services respectifs ou la cellule de crise mise en place dans chacun de ces deux hôpitaux en fonction des besoins.

Pour ce qui concerne les deux SAU, le Tableau 2 indique le personnel médical et paramédical présents normalement dans le SU à $21 \mathrm{~h} 30$ et le personnel présent à $24 \mathrm{~h} 00$ le 13 novembre 2015. À Saint-Antoine, le processus de triage a eu lieu à plusieurs niveaux : un médecin des urgences assu-

\begin{tabular}{|c|c|c|}
\hline & Saint-Louis & Saint-Antoine \\
\hline \multicolumn{3}{|l|}{ Présence sur SU à $21 \mathrm{~h} 15$} \\
\hline IDE & 4 & 8 \\
\hline AS & 3 & 7 \\
\hline Urgentiste & 2 & 4 \\
\hline Cadre & 0 & 1 \\
\hline Personnel administratif & 0 & 1 \\
\hline \multicolumn{3}{|l|}{ Présence sur SU à $24 \mathrm{~h} 00$} \\
\hline IDE & 6 & 15 \\
\hline AS & 5 & 13 \\
\hline Urgentiste & 5 & 8 \\
\hline Cadre & 3 & 4 \\
\hline Personnel administratif & 0 & 2 \\
\hline
\end{tabular}

rait le tri initial des patients, puis à la fin de la période de prise en charge initiale, un anesthésiste-réanimateur permettait de faire le lien permanent avec la SSPI et ainsi coordonner la répartition vers les blocs opératoires. À Saint-Louis, après une phase initiale de triage par un urgentiste en zone d'accueil, un médecin réanimateur médical a été posté sur le parking des urgences pour effectuer le triage à l'arrivée des véhicules de secours. La coordination avec le bloc opératoire a été effectuée avec l'aide d'un chirurgien aux urgences qui se mettait en rapport avec un anesthésiste-réanimateur référent en SSPI. Sur les deux sites, des blocs opératoires supplémentaires ont été immédiatement activés, quatre sur Saint-Antoine, en plus des deux blocs normalement disponibles au cours de la période de garde (trois consacrés à l'orthopédie et trois à la chirurgie viscérale). À Saint-Louis quatre blocs opératoires ont été activés avec la possibilité d'intervenir sur deux salles supplémentaires en cas de besoin. Sur les deux sites, les services de radiologie ont mis en route leur deuxième scanner. Ainsi, 25 scanners ont pu être réalisés entre $22 \mathrm{~h} 00$ et $5 \mathrm{~h} 00$ à Saint-Antoine (la moyenne habituelle pendant cette période étant de 7) et 14 à Saint-Louis. En parallèle, dès le début du déclenchement du plan de crise, des lits ont été réouverts dans des unités d'hôpitaux de semaine, fermées le vendredi soir ( 3 unités, soit 24 lits sur Saint-Antoine, 1 unité de 6 lits sur Saint-Louis) ou des unités de chirurgie ambulatoire. Une cellule d'urgence médicopsychologique a été ouverte dès $23 \mathrm{~h} 00$ à Saint-Antoine par le psychiatre de garde dans une salle située à 50 mètres du $\mathrm{SU}$, elle a permis de prendre en charge 33 personnes proches des patients ou présentes sur les sites d'attaque sans lésions par balles. 


\section{Éléments de dysfonctionnement soulevés et pistes d'amélioration}

Les éléments de difficulté de fonctionnement repérés lors des réunions de retour d'expérience effectuées dans chaque site sont indiqués ci-dessous ainsi que les propositions d'amélioration correspondant à chacun de ces éléments :

- élément 1 : difficultés d'évaluation de la gravité de la situation par les premiers messages d'alerte transmis par les témoins ou les impliqués dans un contexte où l'alerte de proximité précède le message institutionnel (décision de déclenchement du Plan Blanc);

- proposition 1 : améliorer si possible la prise de renseignement auprès des lanceurs d'alerte et croiser les informations avec le Samu et la direction de l'hôpital ;

- élément 2 : difficulté initiale d'assurer le tri UA/UR et la prise en charge des patients graves ;

- proposition 2 : désignation le plus précocement possible d'un médecin urgentiste expérimenté (dès l'arrivée du premier renfort d'un médecin urgentiste) dévolu au tri, à l'orientation des patients dans les différentes zones du SU et qui soit l'interlocuteur du médecin de coordination de la salle de réveil et des blocs opératoires (pour les patients identifiés comme UA) ;

- élément 3 : adapter le tri devant un événement exceptionnel (ici, nombre élevé de patients blessés par balles) ;

- proposition 3: apport substantiel de la présence d'un orthopédiste expérimenté pour le tri des blessés par balles relevant d'un geste de chirurgie orthopédique ; ce tri classait les malades en trois niveaux : T1 : intervention dès que possible, $\mathrm{T} 2:$ hospitalisation avec surveillance étroite du membre et intervention prévue après opération des $\mathrm{T} 1$ ou transfert si délai supérieur à 12 heures; T3 : intervention pouvant être différée au lendemain ;

- élément 4 : difficulté pour joindre les personnels à partir du listing global de l'hôpital ;

- proposition 4 : organisation d'une mobilisation des personnels au niveau de chaque service à partir d'un ou de plusieurs référents alertés par la cellule de crise en notant le rôle majeur des cadres au niveau des services qui ont la meilleure connaissance en temps réel de la disponibilité des personnels (proximité, relève, congés, absence...). Suggestion d'un appel par SMS groupé. Nécessité d'évaluation de l'apport des réseaux sociaux ;

- élément 5 : nécessité d'assurer l'accueil et le traitement des patients consultant aux urgences sans rapport avec l'attaque terroriste ;

- proposition 5 : consacrer l'activité des médecins ne travaillant habituellement pas aux urgences (ressources internes disponibles puis médecins venus spontanément) à la prise en charge des patients relevant d'une consultation sans décompensation aiguë, ne présentant pas de signe prédictif d'une acutisation et nécessitant éventuellement des examens complémentaires (CCMU $1,2$ et 3$)$;

- élément 6 : difficultés de gestion de l'inscription informatique des patients en cas d'afflux massif;

- proposition 6 : prévoir rapidement un renfort en personnel et des modalités d'inscription simplifiées (en mode « dégradé »);

- élément 7 : difficultés d'identification des patients dont certains sont venus spontanément et d'autres régulés et préidentifiés par le Samu ;

- proposition 7 : prévoir un système d'identification propre au service mais spécifique à un plan d'urgence et en cohérence avec le système d'identification préhospitalier;

- élément 8 : difficultés pour maintenir à jour le tableau informatique des urgences en temps réel (positionnement et sortie des patients du SAU) ;

- proposition 8 : désignation d'un médecin, responsable des sorties qui s'assure de l'intégrité du dossier des sortants du SU, contrôle les prescriptions, la destination des patients (équipe d'accueil prévenue) et qui assure la mise à jour du tableau informatique des urgences ;

- élément 9 : difficultés pour obtenir des informations sur le nombre de patients potentiels et leur gravité et le moment de l'arrêt des flux arrivant, l'ensemble ayant été vécu trop passivement ;

- proposition 9: une information au niveau de la cellule de crise doit être organisée en intégrant les données recueillies auprès de la régulation du Samu et les données apportées par les médias qui sont souvent plus aisément disponibles que celles des professionnels sur le terrain absorbés par leur activité. Prévoir un lien régulier d'information entre la cellule de crise de chaque site et le SU pour pallier cet isolement ;

- élément 10 : difficultés liées à la sécurité.

Hôpital considéré comme lieu de refuge par les impliqués, comme source de renseignement pour les familles, voire comme lieu d'investigation pour des journalistes. Il est à noter que les réunions de débriefing dans les deux sites n'ont pas fait apparaître de craintes particulières des personnels pour leur sécurité pendant l'événement.

Sécurité devant permettre l'accès aux patients et aux personnels de l'hôpital ;

- proposition 10 : nécessité de prévenir très précocement le responsable de la sécurité de l'événement, de l'ampleur prévisible et du type d'événement; privilégier le contrôle à l'entrée de l'hôpital qu'à l'entrée des urgences, assurer cependant une présence de la sécurité au SAU permettant si nécessaire d'écarter les personnes non désirables ; développer l'utilisation des cartes professionnelles pour faciliter l'accès à l'hôpital des personnels ;

- élément 11 : nécessité de mobiliser rapidement du matériel adapté au type d'événement; les armoires prédéfinies 
dans le cadre du Plan Blanc du site ne correspondent pas toujours au besoin lié à l'événement matériel ;

- proposition 11 : utilisation des dotations spécifiques (armoire de stockage Plan Blanc) dont la localisation doit être connue des cadres des urgences. Adaptation nécessaire à l'événement avec la nécessité d'une alerte rapide de la pharmacie de l'hôpital ;

- élément 12 : nécessité de transfusion importante en culots globulaires, plasma frais congelé et concentrés plaquettaires. Pas de problème observé d'approvisionnement en produits sanguins. Stock de dispositifs de contrôle de compatibilité prétransfusionnelle insuffisant. Absence de dotation spécifique d'acide tranexamique dans les lots Plan Blanc ;

- proposition 12 : augmentation du stock de dispositifs de contrôle prétransfusionnel et adaptation des dotations thérapeutiques des lots Plan Blanc ;

- élément 13 : difficultés liées aux dysfonctionnements potentiels des applications informatiques couramment utilisées dans le cadre de la prise en charge des patients. Ces dysfonctionnements se sont manifestés par l'impossibilité de consulter à certains moments les documents d'imagerie sur écran informatique et la nécessité de faire graver des cédérom pour accéder aux images ;

- proposition 13 : alerte précoce d'un informaticien pour intervention sur le terrain en cas d'instabilité d'un des systèmes ou d'un des logiciels utilisés ;

- élément 14 : recours à des compétences externes à l'hôpital pour certains patients dont l'arrivée a été spontanée ou imposée (fusillade en cours) ;

- proposition 14 : discussion de l'intérêt comparé du transfert du patient complexe dans ces circonstances ou de la venue sur site de la compétence (exemple : chirurgie vasculaire) ;

- élément 15 : difficulté de gestion des appels pour obtenir des informations sur les patients accueillis ou éventuellement accueillis ;

- proposition 15 : mise en place précoce d'un délégué à la réception des appels sur lequel convergent les demandes de renseignement ;

- élément 16 : difficulté à prendre en charge les accompagnants en état de stress ;

- proposition 16 : mise en place rapide d'une CUMP avec renfort d'au moins un psychiatre afin d'assurer également la prise en charge des malades psychiatriques du flux habituel des urgences.

\section{Discussion}

Lors d'un événement, tels que les attentats terroristes du 13 novembre 2015 à Paris et au Stade de France, l'ensemble du système hospitalier est amené à s'adapter en fonction de l'importance du nombre de victimes et de leur gravité [3-5]. Les hôpitaux à proximité de l'événement jouent un rôle particulier dans la mesure où ils peuvent être le lieu d'un afflux massif de victimes dont la plupart viennent spontanément [6]. L'analyse de l'activité des hôpitaux de Saint-Louis et de Saint-Antoine, la nuit du 13 au 14 novembre 2015, montre que si l'impact quantitatif a été relativement contrôlé, la gravité des patients et le type de lésions, nécessitant toutes un acte chirurgical, représentait un défi organisationnel. Contrairement aux autres établissements sollicités de la région et ayant reçus les UA directement en SSPI, tous les patients arrivés dans les hôpitaux de proximité sont passés par le SU. Une des particularités du contexte parisien est le nombre important d'établissements pouvant accueillir des urgences lourdes. Cela a permis à la régulation du Samu de Paris d'orienter la plupart des urgences triées «UA » sur les sites des attentats, vers d'autres établissements, épargnant relativement ainsi ces deux hôpitaux de proximité. Le recours aux SU de proximité a été largement utilisé par les premiers secours non régulés. On peut noter que malgré le nombre total de patients accueillis sur les deux sites, le nombre de patients présents dans les urgences a décru rapidement grâce à la décision dès $22 \mathrm{~h}$ d'admettre en hospitalisation standard tous les patients hospitalisés dans la journée en UHCD et les patients en attente d'hospitalisation et par le départ accéléré des patients vus dans la journée grâce au renfort médical. Pour Saint-Antoine, il n'a jamais atteint le niveau constaté au maximum d'activité, soit le 13 novembre 2015 à 17 h30 (Fig. 2).

La nature des victimes était majoritairement des patients présentant de plaies par balles dont plus des deux tiers étaient venus par ambulances ou par véhicule de secours non régulés par le Samu. Dans de telles circonstances, on constate que l'alerte a été provoquée par l'arrivée des premiers patients, qu'elle a entraîné le déclenchement d'un plan d'urgence au sein de l'hôpital et l'alerte des autorités de l'événement. Le faible volume de patients arrivés debout n'a pas entraîné l'ouverture d'un lieu spécifique différent de celui de l'accueil des urgences. Le triage à l'entrée des urgences, par un médecin expérimenté, en plusieurs niveaux définis en fonction des zones aux urgences, est un élément fondamental inscrit dans le Plan Blanc. Les spécificités liées à la pathologie balistique ont peu modifié le processus de prise en charge des patients au niveau du SU. Ce processus impliquant une évaluation initiale, la mise en route du traitement et l'orientation répond à des critères proches de ceux utilisée pour les traumatisés consultants habituellement aux urgences y compris dans la filière SOS mains de l'hôpital Saint-Antoine. L'aide d'un orthopédiste pour le tri des patients, après stabilisation et prise en charge initiale, est à souligner comme un élément majeur dans l'organisation du SU. 
Si la prise en charge de patients blessés par balles répond globalement au même processus de prise en charge des patients traumatisés, certaines spécificités peuvent cependant être notées pour adapter les organisations comme la possibilité de « taylorisation » de certaines tâches répétitives et les stocks avec la nécessité d'un nombre important [7] :

- de poses de voies veineuses périphériques avec prélèvement de bilans préopératoires ;

- de solutés de remplissage et de transfusions ;

- de traitements injectables (morphiniques, antibiotiques, acide tranexamique...) [8];

- de pansements compressifs et de grands pansements de protection;

- de recours à l'imagerie et particulièrement de scanners.

La problématique d'un hôpital de proximité, dans les circonstances particulières d'un attentat terroriste par balles, est essentiellement liée aux capacités d'adaptation pour accueillir les nombreux patients [9]. L'augmentation de la capacité d'accueil a pu être possible grâce à l'ouverture sans délai d'unités d'hospitalisation qui ont permis d'assurer l'aval des patients des SU incluant l'UHCD, la mobilisation des équipes permettant la suite de la prise en charge des malades (réanimateurs médicaux, anesthésistes-réanimateurs, personnels de bloc, radiologues, psychiatres, personnels administratifs et logistiques) (Fig. 4).

On constate que l'implication des deux SU dans ce processus a été intense mais aussi relativement limitée dans le temps (une douzaine d'heures). Il a donc fallu dans cet intervalle s'adapter à l'ampleur de la situation en associant à la fois plus de vitesse et de la démultiplication (« changement

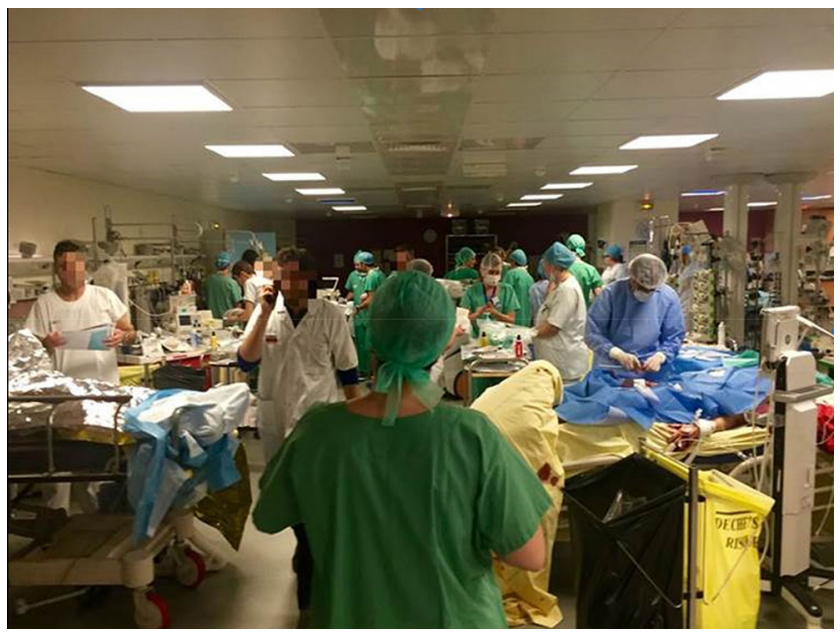

Fig. 4 Salle de surveillance postinterventionelle (SSPI) de l'hôpital Saint-Louis, nuit du 13 au 14 novembre 2015. Crédit photo (C) Pourya Pashootan/AP-HP de braquet ») [10]. Le lendemain, l'activité des deux services a été une des plus faibles de l'année.

\section{Conclusion}

Les hôpitaux à proximité d'un événement exceptionnel avec de multiples victimes, tel que les actes terroristes que Paris a connus, sont sollicités de façon particulière, et chaque hôpital doit s'y préparer. Il est essentiel que la régulation médicale du Samu prenne en compte la situation spécifique de ces hôpitaux et les préserve autant que possible de l'arrivée de patients régulés, car de nombreux patients parfois graves viennent spontanément vers ces hôpitaux. Le retour d'expérience est une étape permettant de faire émerger des pistes d'amélioration. L'expérience parisienne reste cependant singulière du fait de la densité d'établissements dans la région Île-de-France [7]. La coopération de l'ensemble des structures impliquées dans la prise en charge des malades est un élément fondamental d'efficacité du plan d'Urgence et la solidarité manifestée lors de l'événement ainsi que les compétences professionnelles ont permis un bon fonctionnement du service des urgences. La capacité des SAU à gérer au quotidien la prise en charge de patients en évaluant leur gravité et leur stabilité, tout en s'adaptant en permanence aux conditions de flux et de capacité d'hospitalisation, positionne ces structures comme un maillon opérationnel essentiel de la prise en charge des victimes d'attentats dans un contexte d'afflux massif. Les SU ont pu s'appuyer sur des liens tissés au quotidien avec les différentes équipes pour s'adapter à cette catastrophe. L'importance du Plan Blanc, de sa connaissance, de son application et de son actualisation doit être soulignée, tout comme la formidable mobilisation des personnels et des structures. Cet ensemble a permis aux équipes de s'adapter et de maitriser la situation en faisant rapidement appel aux forces déjà présentes dans l'hôpital, alors même que le Plan Blanc n'avait pas encore été officiellement déclenché, permettant une mise en place immédiate de circuits exceptionnels. Dans ce contexte hors du commun, aucune compromission n'a été faîtes sur la qualité des soins qui ont été prodigués aux nombreuses victimes. Si l'implication des urgentistes pour la prise en charge directe des victimes se compte en heures, celle des chirurgiens a été de quelques jours et celui des psychiatres et des rééducateurs durera plusieurs mois.

Remerciements Les auteurs remercient le Dr Dominique Brun-Ney et Laure Beaujouan de la direction de l'organisation médicale et des relations avec les universités de l'APHP de leur avoir fourni la Figure 1.

Liens d'intérêts : les auteurs déclarent ne pas avoir de lien d'intérêt. 


\section{Références}

1. Direction de l'hospitalisation et de l'organisation des soins (DHOS) (2002) Circulaire DHOS/HFD no 2002/284 du 3 mai 2002 relative à l'organisation du système hospitalier en cas d'afflux de victimes. www.sante.gouv.fr/IMG/pdf/circulaire_du_2_mai_2003-2.pdf (dernier accès le 2 décembre 2015)

2. Ministère de la Santé, de la Famille et des Personnes handicapées (2002) Explosion de l'usine AZF de Toulouse le 21 septembre 2001. Rapport de mission, enseignement et propositions sur l'organisation des soins. Septembre 2002. http://www.ladocumentationfrancaise.fr/var/storage/rapports-publics/034000001.pdf (dernier accès le 2 décembre 2015)

3. Okumura T, Suzuki K, Fukuda A, et al (1998) The Tokyo subway sarin attack: disaster management, Part 2: Hospital response. Acad Emerg Med 6:618-24

4. Gates JD, Arabian S, Biddinger P, et al (2014) The initial response to the Boston marathon bombing: lessons learned to prepare for the next disaster. Ann Surg 260:960-66

5. Aylwin CJ, König TC, Brennan NW, et al (2006) Reduction in critical mortality in urban mass casualty incidents: analysis of triage, surge, and resource use after the London bombings on July 7, 2005. Lancet 368:2219-25

6. Gutierrez de Ceballos JP, Turégano Fuentes F, et al (2005) Casualties treated at the closest hospital in the Madrid, March 11, terrorist bombings. Crit Care Med 33:S107-S12

7. Hirsch M, Carli P, Nizard R, et al (2015) The medical response to multisite terrorist attacks in Paris. Lancet 368:2535-8

8. CRASH-2 trial collaborators, Shakur H, Roberts I, et al (2010) Effects of tranexamic acid on death, vascular occlusive events, and blood transfusion in trauma patients with significant haemorrhage (CRASH-2): a randomized, placebo-controlled trial. Lancet 376:23-32

9. Ministère de la santé et des solidarités (2006) Guide d'aide à l'élaboration des plans blancs élargis et des plans blancs des établissements. Édition 2006, annexe à la circulaire $\mathrm{n}^{\mathrm{o}} \mathrm{DHOS} / \mathrm{CGR} / 2006 / 401$ du 14 septembre $2006 \mathrm{http} / /$ www. sante.gouv.fr/IMG/pdf/plan_blanc_2006.pdf (dernier accès le 2 décembre 2015)

10. Virenque C (2005) Le Plan Blanc : afflux massif de victimes. Réanimation $14: 712-5$ 